\title{
X-ray spectroscopy and photometry of the long-period polar Al Trianguli with XMM-Newton ${ }^{\star}$
}

\author{
I. Traulsen ${ }^{1}$, K. Reinsch ${ }^{1}$, R. Schwarz ${ }^{2}$, S. Dreizler ${ }^{1}$, K. Beuermann ${ }^{1}$, A. D. Schwope ${ }^{2}$, and V. Burwitz ${ }^{3}$ \\ 1 Institut für Astrophysik, Georg-August-Universität Göttingen, Friedrich-Hund-Platz 1, 37077 Göttingen, Germany \\ e-mail: traulsen@astro.physik.uni-goettingen.de \\ 2 Astrophysikalisches Institut Potsdam, An der Sternwarte 16, 14482 Potsdam, Germany \\ 3 Max-Planck-Institut für extraterrestrische Physik, Postfach 1312, 85741 Garching, Germany
}

Received 28 August 2009 / Accepted 6 March 2010

\section{ABSTRACT}

\begin{abstract}
Context. The energy balance of cataclysmic variables with strong magnetic fields is a central subject in understanding accretion processes on magnetic white dwarfs. With XMM-Newton, we perform a spectroscopic and photometric study of soft X-ray selected polars during their high states of accretion.

Aims. On the basis of X-ray and optical observations of the magnetic cataclysmic variable AI Tri, we derive the properties of the spectral components, their flux contributions, and the physical structure of the accretion region in soft polars.

Methods. We use multi-temperature approaches in our XSPEC modeling of the X-ray spectra to describe the physical conditions and the structures of the post-shock accretion flow and the accretion spot on the white-dwarf surface. In addition, we investigate the accretion geometry of the system by completing a timing analysis of the photometric data.

Results. Flaring soft X-ray emission from the heated surface of the white dwarf dominates the X-ray flux during roughly $70 \%$ of the binary cycle. This component deviates from a single black body and can be described by a superimposition of mildly absorbed black bodies with a Gaussian temperature distribution between $k T_{\mathrm{bb}, \text { low }}:=2 \mathrm{eV}$ and $k T_{\mathrm{bb} \text {,high }}=43.9_{-3.2}^{+3.3} \mathrm{eV}$, and $N_{\mathrm{H}, \mathrm{ISM}}=1.5_{-0.7}^{+0.8} \times 10^{20} \mathrm{~cm}^{-2}$. In addition, weaker hard X-ray emission is visible nearly all the time. The spectrum from the cooling post-shock accretion flow is most closely fitted by a combination of thermal plasma MEKAL models with temperature profiles adapted from prior stationary two-fluid hydrodynamic calculations. The resulting plasma temperatures lie between $k T_{\text {MEKAL,low }}=0.8_{-0.2}^{+0.4} \mathrm{keV}$ and $k T_{\text {MEKAL,high }}=20.0_{-6.1}^{+9.9} \mathrm{keV}$; additional intrinsic, partial-covering absorption is on the order of $N_{\mathrm{H}, \mathrm{int}}=3.3_{-1.2}^{+2.5} \times 10^{23} \mathrm{~cm}^{-2}$. The soft X-ray light curves show a dip during the bright phase, which can be interpreted as self-absorption in the accretion stream. Phase-resolved spectral modeling supports the picture of one-pole accretion and self-eclipse. One of the optical light curves corresponds to an irregular mode of accretion. During a short XMM-Newton observation at the same epoch, the X-ray emission of the system is clearly dominated by the soft component.
\end{abstract}

Key words. novae, cataclysmic variables - stars: individual: AI Tri - X-rays: binaries - accretion, accretion disks

\section{Introduction}

AI Tri (RX J0203.8+2959) was first described within a sample of ROSAT-discovered bright soft X-ray sources by Beuermann \& Thomas (1993). Their classification of AI Tri as an AM Her type binary (also called a polar) was later confirmed in a multiwavelength study by Schwarz et al. (1998), who identified cyclotron humps in optical spectra obtained during a high state of accretion. The orbital period of $P_{\text {orb }}=4.6 \mathrm{~h}$ is one of the longest known among polars, whereas the magnetic field strength of $B=38 \pm 2 \mathrm{MG}$ and the amplitude of the long-term brightness variations between $V=18.0-15 \cdot 5$ (Schwarz et al. 1998) lie in the typical parameter range of this class. Based on the wavelength dependence of the UBVRI light curve minima and the variations in both the linear and the circular polarization, Katajainen et al. (2001) suggest that the system has a high inclination of $i \approx 70^{\circ} \pm 20^{\circ}$ and accretes onto two almost equally fed magnetic poles. On the other hand, Schwarz et al. (1998) propose that a single dominating accretion region is active at the epoch of their observations.

* Based on observations obtained with XMM-Newton, an ESA science mission with instruments and contributions directly funded by ESA Member States and NASA.
AI Tri belongs to a significantly large group of AM Her systems that were found to emit almost entirely at X-ray energies below $0.5 \mathrm{keV}$ during the ROSAT all-sky survey (Beuermann \& Thomas 1993; Thomas et al. 1998; Beuermann et al. 1999). Although these systems could play an important role in investigating the energy balance of polars, only a few of them have been studied using high-resolution X-ray spectroscopy (Ramsay \& Cropper 2003; Ramsay et al. 2004). We, therefore, initiated dedicated observations with XMM-Newton to perform a detailed study of the spectral components, their flux contributions, and the physical structure of the accretion region of polars selected by their distinct soft X-ray fluxes during high-states of accretion. In the following, we present an analysis of the magnetic cataclysmic variable AI Tri based on new XMM-Newton and optical data, and archival ROSAT data.

\section{Observations and data reduction}

\subsection{X-ray observations}

With XMM-Newton, we obtained a $20 \mathrm{ks}(5.6 \mathrm{~h})$ exposure during a high state of AI Tri on August 22, 2005 (observation ID 0306841001), which covers for the first time uninterruptedly more than one complete binary orbit. The EPIC instruments 


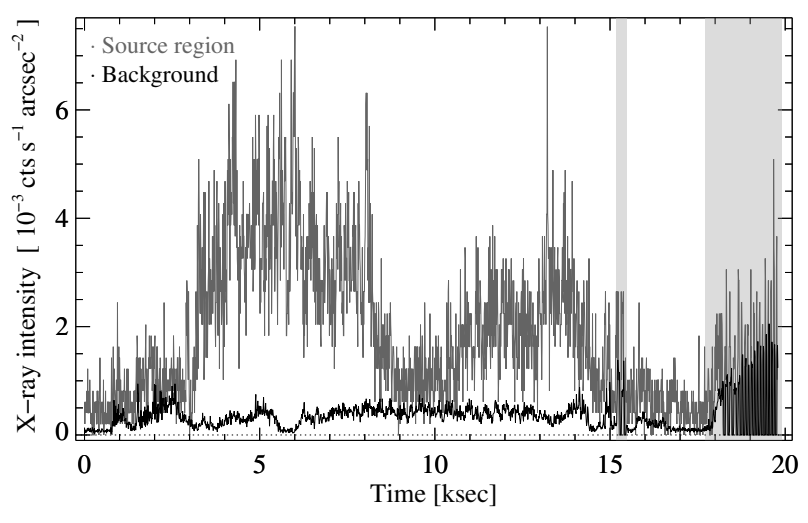

Fig. 1. EPIC/pn X-ray intensity in both the source region (source plus background) and the source-free background region during the $20 \mathrm{ks}$ XMM-Newton observation, binned into time intervals of $10 \mathrm{~s}$.

were operated in full frame mode with the thin filter. The UV light curve at an effective wavelength of $2310 \AA$ was measured simultaneously using the optical monitor $(\mathrm{OM})$ in fast mode with the UVM2 filter. RGS spectra were not used from this pointing, since the X-ray flux of the object was too low, and the net source count rate measured by the RGS instruments was consistent with zero for more than $80 \%$ of the exposure time.

Data were reduced using the XMM-Newton software analysis system SAS v8.0. Large parts of the exposure were affected by an enhanced background signal. We omitted two time intervals from the analysis (Fig. 1): a strong soft proton flare after $15 \mathrm{ks}$ and the last $2 \mathrm{ks}$ of the observation, when the satellite approaches the Van Allen radiation belt. During these intervals, a considerable spectral hardening at energies above $3 \mathrm{keV}$ and spectral softening at energies below $1 \mathrm{keV}$ were detected.

When extracting light curves, spectra, and images, single and double events were processed in the case of EPIC/pn data, supplemented by quadruple events in EPIC/MOS data. Phaseresolved spectra were generated during the four phase intervals defined in Sect. 3. Source data were collected from an aperture of 25 arcsec around the source center, background data from a larger source free region close to the source position. To diminish potential pile-up effects, we excluded the innermost 5 arcsec of the source region when extracting the mean EPIC/pn spectrum and the bright-phase spectra, where the peak count rates (source plus background) reach 6-8 $\mathrm{cts} \mathrm{s}^{-1}$ for EPIC/pn and 3-4 $\mathrm{cts} \mathrm{s}^{-1}$ for EPIC/MOS, respectively. Appropriate spectral response matrices were generated for the individual instruments and extraction regions applying calibration files from the July 2008 release. Spectral bins comprise a minimum of 20 cts (mean EPIC/pn spectrum: $30 \mathrm{cts}$ ). Photon arrival times were corrected to the barycenter of the solar system via the BARYCEN task.

In the soft energy regime below $0.9 \mathrm{keV}$, where the calibration is known to be least precise (Stuhlinger et al. 2008; Mateos et al. 2009), we use the isolated neutron star RX J1856.4-3754 to calibrate the EPIC spectra. RXJ1856.4-3754 was established as a low-energy calibration target for missions such as Chandra, Suzaku, Swift, and XMM-Newton ${ }^{1}$, and exhibits a high soft X-ray and low optical flux. Its spectrum is free from prominent line features and described well by two black bodies (Beuermann et al. 2006). We compare archival XMM-Newton EPIC data of RXJ1856.4-3754, which span more than five years, with the model spectra of Beuermann et al. (2006) and

\footnotetext{
1 International Astronomical Consortium for High Energy Calibration, http: //www . iachec . org
}

carefully adjust the instrumental effective areas. In this approach, we assume that the energy redistribution is independent of the incident energy when considering only objects with very soft $\mathrm{X}$-ray spectra. The discrepancies between data and model remain mostly below 5\%, with larger excesses up to $20 \%$ for EPIC/MOS2.

A first XMM-Newton observation of AI Tri on August 15, 2005 in the same configuration as described above resulted in partially corrupted data (observation ID 0306840901) due to technical problems. During 1.1 to $5 \mathrm{ks}$ (0.3 to $1.4 \mathrm{~h}$ ), EPIC, RGS (1st order), and OM data are usable. We apply the same data reduction steps as applied to the data acquired on August 22, 2005. With peak count rates of $21 \mathrm{cts} \mathrm{s}^{-1}$ collected by EPIC/pn and $1.4 \mathrm{cts} \mathrm{s}^{-1}$ by the RGS detectors, the source flux was considerably higher than one week later. Therefore, we extended the EPIC/pn aperture to 30 arcsec, excising the innermost 7 arcsec. The EPIC spectra were rebinned to at least 20 counts per bin.

In addition, we used unpublished ROSAT archival data obtained between January 15 and February 5, 1998 with the HRI detector. The total integration time of $35 \mathrm{ks}(9.6 \mathrm{~h})$ consisted of nine observation intervals. The individual light curves were extracted with standard tasks of the EXSAS/MIDAS (Zimmermann et al. 1993) software packages, corrected to the barycenter, and combined to a common light curve profile.

\subsection{Optical data}

At the Göttingen $50 \mathrm{~cm}$, the Potsdam $70 \mathrm{~cm}$, the MoNET/North (Texas) $1.2 \mathrm{~m}$, and the Tübingen $80 \mathrm{~cm}$ telescopes, we carried out optical $V$-band and white-light photometry during ten nights between August 2005 and March 2007. AI Tri was found in high or intermediate high states at estimated brightnesses between $V=17.0$ and 15.5 . For data reduction and differential photometry, we used TRIPP (Schuh et al. 2003) and ESO-MIDAS routines. Light curves were determined using differential aperture photometry. Apparent magnitudes of the system were calculated against the $V$-band magnitudes of the comparison stars USNO-B1.0 1199-0026710 $(V=13.29 \pm 0.05)$ and USNO-B1.0 1199-0026672 $(V=14.62 \pm 0.05)$ determined by Schwarz (1996). Fluxes are given relative to their orbital mean, and flux errors were derived by means of photon statistics. The times were converted to terrestrial time and corrected to the barycenter on the basis of the JPL ephemeris (Standish 1998). An overview of all the data obtained is given in Table 1.

\section{Optical, UV, and X-ray light curves}

\subsection{Optical photometry}

The optical $V$-band light curves of AI Tri (e.g. Fig. 2f) during high and intermediate high states of accretion are largely similar to the data presented by Katajainen et al. (2001) and Schwarz et al. (1998). The orbital modulation deviates from a sinusoidal shape and is slightly asymmetric showing a steeper rise and smoother decline. An irregular pattern of narrow small dips is superimposed on the light curves, one dip recurring at photometric phase zero, which has not been seen in earlier data.

The orbital period of AI Tri has not been known accurately enough to extrapolate the photometric ephemeris given by Schwarz et al. (1998) to the epochs of our observations. Schwarz et al. (1998) established photometric phase zero at the broad minima of the optical high-state light curves, the only recurrent feature that could be identified in the vast majority of the light curves. Using timings of six new $V$-band minima mapped 
Table 1. Log of the ROSAT and XMM-Newton observations and of the optical photometry of AI Tri.

\begin{tabular}{|c|c|c|c|c|c|c|c|}
\hline Date & Telescope $^{a}$ & Instrument & Filter & $t_{\text {exp }}[\mathrm{s}]$ & $t_{\text {cycle }}[\mathrm{s}]$ & Duration $[\mathrm{h}]$ & Observer \\
\hline 1998 Jan. $15-$ Feb. 05 & ROSAT & HRI & $0.1-2.4 \mathrm{keV}$ & & & 9.6 & PI Schwarz \\
\hline 2005 Aug. 15 & XMM-Newton & $\mathrm{EPIC} / \mathrm{pn}$ & $0.1-10 \mathrm{keV}$ & & & 0.3 & PI Reinsch \\
\hline 2005 Aug. 15 & XMM-Newton & EPIC/MOS & $0.1-10 \mathrm{keV}$ & & & 1.4 & PI Reinsch \\
\hline 2005 Aug. 15 & XMM-Newton & $\mathrm{OM}$ & UVM2 & & & 1.1 & PI Reinsch \\
\hline 2005 Aug. 15 & XMM-Newton & RGS & $0.3-2.5 \mathrm{keV}$ & & & 1.3 & PI Reinsch \\
\hline 2005 Aug. 22 & XMM-Newton & EPIC & $0.1-10 \mathrm{keV}$ & & & 5.6 & PI Reinsch \\
\hline 2005 Aug. 22 & XMM-Newton & $\mathrm{OM}$ & UVM2 & & & 5.6 & PI Reinsch \\
\hline 2005 Aug. 17 & AIP $70 \mathrm{~cm}$ & TK1024-01 & $\mathrm{V}$ & 60 & 66 & 6.5 & Schwarz \\
\hline 2005 Aug. 29 & AIP $70 \mathrm{~cm}$ & TK1024-01 & $\mathrm{V}$ & 60 & 66 & 7.6 & Schwarz \\
\hline 2006 Nov. 09 & IAG $50 \mathrm{~cm}$ & STL-6303E & WL & 180 & 191 & 6.6 & Traulsen \\
\hline 2006 Nov. 15 & IAG $50 \mathrm{~cm}$ & STL-6303E & $\mathrm{V}$ & 240 & 251 & 4.7 & Traulsen \\
\hline 2006 Nov. 16 & IAG $50 \mathrm{~cm}$ & STL-6303E & V & 240 & 247 & 1.8 & Traulsen \\
\hline 2007 Jan. 14 & IAG $50 \mathrm{~cm}$ & STL-6303E & $\mathrm{V}$ & $90-180$ & $97-187$ & 2.2 & Traulsen \\
\hline 2007 Jan. 25 & AIP $70 \mathrm{~cm}$ & TK1024-01 & $\mathrm{V}$ & 90 & 95 & 4.6 & Schwarz \\
\hline 2007 Jan. 29 & MONET/North & Alta E47+ & V & 15 & 18 & 1.8 & Hessman \\
\hline 2007 Jan. 30 & MONET/North & Alta E47+ & V & 15 & 18 & 2.0 & Hessman \\
\hline 2007 Mar. 14 & IAG $50 \mathrm{~cm}$ & STL-6303E & $\mathrm{V}$ & 150 & 157 & 1.8 & Traulsen \\
\hline 2007 Mar. 14 & AIT $80 \mathrm{~cm}$ & STL-1001E & V & $20-30$ & $23-33$ & 1.5 & Nagel \\
\hline
\end{tabular}

Notes. ${ }^{(a)}$ AIP: Astrophysikalisches Institut Potsdam. IAG: Institut für Astrophysik Göttingen. AIT: Institut für Astronomie und Astrophysik Tübingen, Abt. Astronomie (Kepler Center for Astro and Particle Physics).

between August 2005 and March 2007 in addition to the data published by Schwarz et al. (1998), we updated their photometric ephemeris. We determined the minima by performing Gaussian fits to the light curves, excluding the dips (Table 2). By means of a least-squares method, the inverse square sum of observed minus calculated minimum times $(\mathrm{O}-\mathrm{C})^{-2}$ was minimized for narrowly spaced trial periods within a $\pm 10 \sigma$ range of the Schwarz et al. (1998) value. We derived the improved $V$-band ephemeris

$\mathrm{BJD}_{\min }(\mathrm{TT})=2451439.0391(10)+0.19174566(9) \times E$,

which we use throughout this paper.

In addition, Schwarz et al. (1998) determined an orbital ephemeris of the system from long-slit spectroscopy. They defined $\varphi_{\text {orb }}=0$ at the blue-to-red zero crossing of the radial velocities, which corresponds to $\varphi_{\text {phot }}=0.191 \pm 0.080$, and identified it with the inferior conjunction of the secondary. A constant relation between photometric and orbital phase can be assumed, if the system rotates synchronously, as most polars do. Schwarz et al. (1998) derived a degree of synchronism better than $\left|P_{\text {orb }}-P_{\text {spin }}\right| / P_{\text {orb }}<10^{-4}$ for AI Tri. We show the orbital phasing as $\varphi_{\text {phot }}-0.191$ at the top of Fig. 2, since it can provide geometrical information about the system (Sect. 6).

\subsection{XMM-Newton light curves}

The observation with the optical monitor and the UVM2 filter at an effective wavelength of $2310 \AA$ presents the first mid ultraviolet light curve of AI Tri (Fig. 2e). It exhibits a roughly similar shape but larger amplitude than the optical light curves, an almost sinusoidal variation, and an additional dip in the light curve minimum near $\varphi_{\text {phot }}=0.05$. This dip is shifted by about $\Delta \varphi=0.05$ in phase relative to the dip within the optical minimum. The UV maximum at $\varphi_{\text {phot }}=0.45$ appears to be flattened, but due to the lack of additional UV data it remains unclear whether this feature recurs or is unique.

From the $20 \mathrm{ks}$ exposure performed by the EPIC instruments, we extracted the first continuous X-ray light curves of AI Tri over a full binary orbit (Figs. 2b, c). Two X-ray bright phases
Table 2. Barycentric timings of the $V$-band minima of AI Tri.

\begin{tabular}{ccrr}
\hline \hline $\mathrm{BJD}_{\min }(\mathrm{TT})$ & $\Delta T_{\min }$ & \multicolumn{1}{c}{$\mathrm{O}-\mathrm{C}$} & \multicolumn{1}{c}{ Cycle } \\
\hline $2449243.93702^{a}$ & 0.0008 & 0.0114 & -11448 \\
$2450042.36390^{a}$ & 0.0017 & 0.0008 & -7284 \\
$2450043.51363^{a}$ & 0.0009 & -0.0031 & -7278 \\
$2450046.39803^{a}$ & 0.0029 & 0.0398 & -7263 \\
$2450047.54570^{a}$ & 0.0014 & 0.0252 & -7257 \\
$2450049.26491^{a}$ & 0.0013 & -0.0087 & -7248 \\
$2450049.45553^{a}$ & 0.0011 & -0.0146 & -7247 \\
$2450122.32708^{a}$ & 0.0019 & 0.0281 & -6867 \\
$2450123.28113^{a}$ & 0.0013 & 0.0037 & -6862 \\
$2450423.36107^{a}$ & 0.0009 & -0.0068 & -5297 \\
$2450748.74298^{a}$ & 0.0047 & -0.0613 & -3600 \\
$2450748.95113^{a}$ & 0.0063 & 0.0242 & -3599 \\
2453612.47355 & 0.0003 & -0.0133 & 11335 \\
2454055.59847 & 0.0014 & -0.0096 & 13646 \\
2454115.23645 & 0.0006 & 0.0169 & 13957 \\
2454126.35745 & 0.0007 & 0.0156 & 14015 \\
2454129.62165 & 0.0008 & 0.0392 & 14032 \\
2454174.27428 & 0.0072 & -0.0865 & 14265 \\
\hline
\end{tabular}

Notes. ${ }^{(a)}$ Schwarz et al. (1998).

during the intervals $\varphi_{\text {phot }}=0.05-0.40$ and $\varphi_{\text {phot }}=0.50-0.80$, as defined by the ephemeris given above, commence with a steep rise at $\varphi_{\text {phot }}=0.05$. They are interrupted by a short soft minimum at $\varphi_{\text {phot }}=0.40-0.50$ and a larger interval of low flux between $\varphi_{\text {phot }}=0.80$ and 1.05 (faint phase). The flux variation is most pronounced in the soft energy band, the bright phases lasting about $70 \%$ of the orbital period, while hard X-ray emission remains visible at a low level at all times. The hard X-ray flux increases by a factor of about three at the beginning of the first bright soft phase and varies slightly during the second one.

The hardness ratio $\mathrm{HR}=(\mathrm{H}-\mathrm{S}) /(\mathrm{H}+\mathrm{S})$, where $\mathrm{H}$ and $\mathrm{S}$ represent the counts at energies above and below $0.5 \mathrm{keV}$, respectively, correlates largely with the light curves in the soft energy band, and tends to +1 during faint and to -1 during bright phases (Fig. 2d). Throughout the soft minimum at $\varphi_{\text {phot }}=0.45$, the hardness ratio levels off around 0 and thus differs from the faint-phase behavior, where it rises to values around 0.7. 


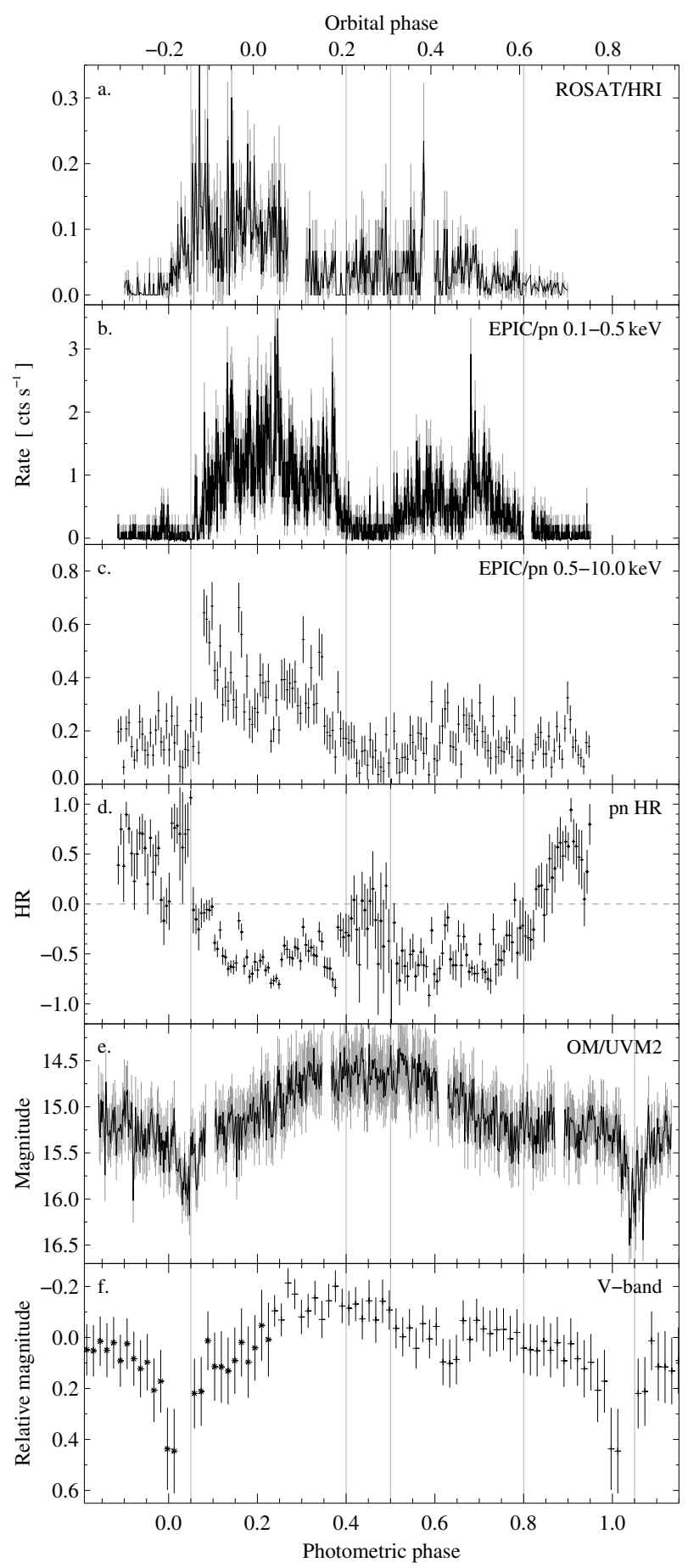

Fig. 2. X-ray, ultraviolet, and optical light curves of AI Tri folded over photometric phase. Orbital phase as $\varphi_{\text {orb }}=\varphi_{\text {phot }}-0.191$ according to Schwarz et al. (1998) is marked at the top of the plot. a) ROSAT/HRI data of Jan./Feb. 1998 with a total integration time of $35 \mathrm{ks}$, combined to a common light curve profile and rebinned into $30 \mathrm{~s}$ intervals. b)-d) EPIC/pn light curve on August 22, 2005, divided into the soft component $\mathrm{S}$, the hard component $\mathrm{H}$, and the associated hardness ratio $\mathrm{HR}=(\mathrm{H}-\mathrm{S}) /(\mathrm{H}+\mathrm{S})$. The soft band data have been binned into $10 \mathrm{~s}$, the hard band and the hardness ratio into $100 \mathrm{~s}$. e) OM light curve in the ultraviolet UVM2 filter at an effective wavelength of $2310 \AA$ in time bins of $30 \mathrm{~s}$. f) Optical $V$-band data obtained at the Göttingen $50 \mathrm{~cm}$ telescope on November 15, 2006, plotted twice for clarity.

Similar characteristics of the light curves are found from the ROSAT/HRI data between January 15 and February 5, 1998 (Fig. 2a).

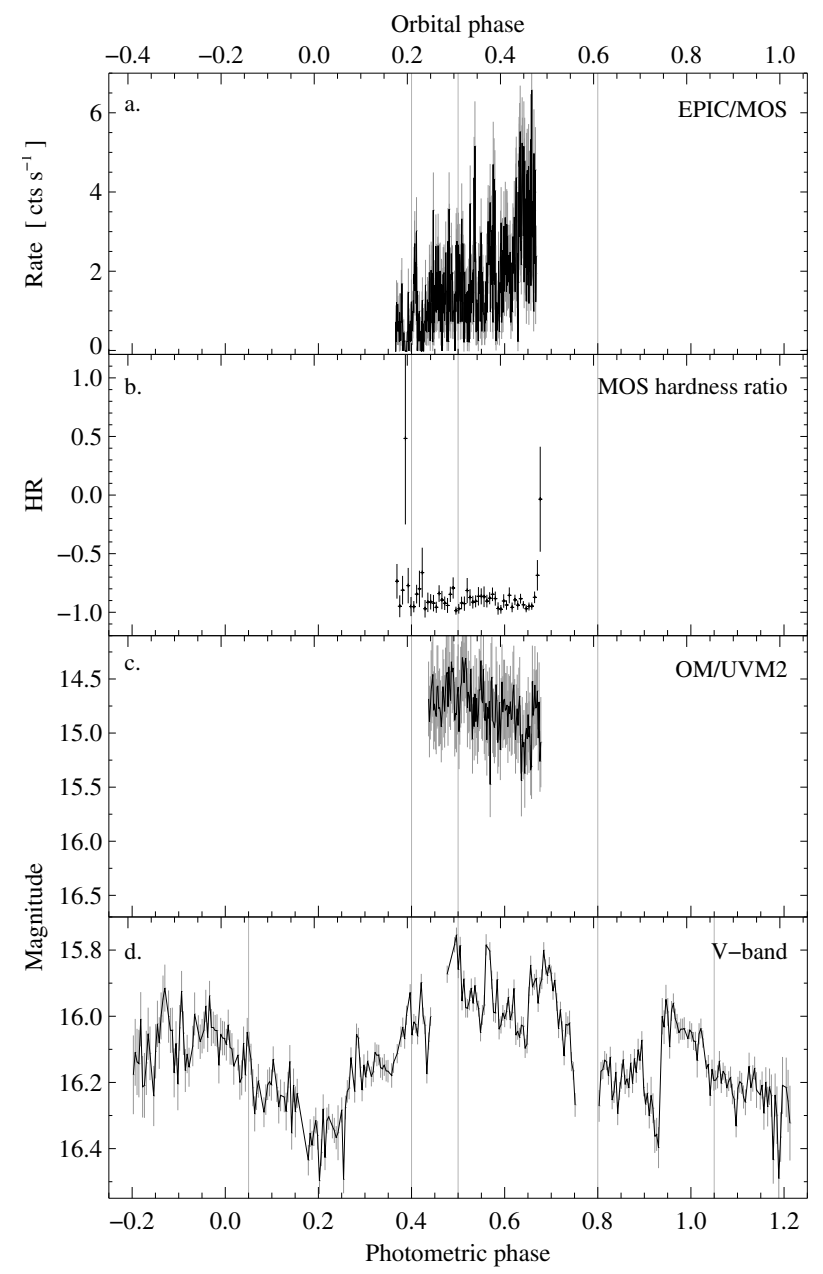

Fig. 3. Multi-wave-band light curves obtained with XMM-Newton on Aug. 15 (a)-c)) and with the Potsdam $70 \mathrm{~cm}$ telescope on Aug. 17, 2005 (d)). The X-ray data are binned into $10 \mathrm{~s}$ intervals, the ultraviolet into $30 \mathrm{~s}$, and the hardness ratios into $100 \mathrm{~s}$, respectively. Grey lines indicate the phases of high and low soft X-ray flux in the $20 \mathrm{ks}$ exposure.

\subsection{Irregular mode on August 17, 2005}

The $V$-band light curve on August 17, 2005 (Fig. 3d) differs markedly from the majority. At a magnitude around $V=16.0$ (close to that during high states of accretion), the light curve is strikingly variable and more asymmetric than in normal high states; the photometric minimum appears to be shifted by 0.2 in phase. In consequence, we did not include this peculiar light curve in the determination of the new ephemeris. During the next observation on August 29, the $V$-band light curve fits in the prevailing shape again. Schwarz et al. (1998) described a comparable phase-shift with an altered light curve shape in their $R V$ photometry of October and November 1992 as an "irregular mode".

The irregular light curve was observed only two days after the curtailed XMM-Newton exposure on August 15, 2005 (Fig. 3a-c). The EPIC/MOS and RGS1 data cover phases $\varphi_{\text {phot }}=$ $0.35-0.65$ and EPIC/pn $\varphi_{\text {phot }}=0.58-0.65$, respectively. This is equivalent to the soft minimum phase and about the first half of the second bright phase of the August 22 light curves. The EPIC count rate in the soft energy band, marked by pronounced flickering, is about a factor of eight higher than one week later. In contrast, little hard X-ray emission was registered, not exceeding the low level of hard X-rays on August 22. The hardness ratios remain almost constant at about -0.87 , even during 


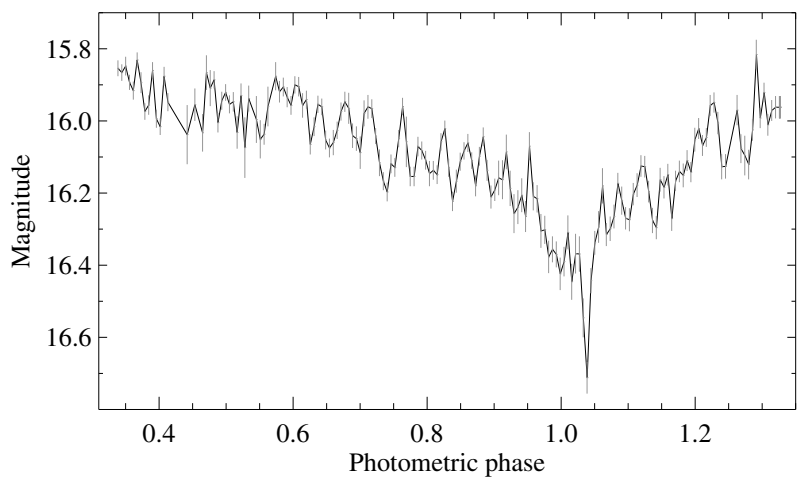

Fig. 4. $V$-band light curve acquired on January 25, 2007 at the $70 \mathrm{~cm}$ telescope of the Astrophysical Institute Potsdam. Cycle time is $100 \mathrm{~s}$.

the $\varphi_{\text {phot }}=0.4-0.5$ interval, where they increase to zero in the August 22 data. The ultraviolet light curve has similar magnitudes as on August 22. Owing to the insufficient coverage of the orbital cycle, the appearance of the soft X-ray minimum and a potential phase shift cannot be determined.

\subsection{Short-period variations}

The multiwavelength light curves are highly variable and exhibit, on timescales as short as one minute, strong and potentially recurring flickering, which is typical of soft polars. Schwarz et al. (1998) describe possible quasi-periodic oscillations (QPOs) at 6.5-7 $\mathrm{min}$ and $13.5-14 \mathrm{~min}$ in their optical data. We examine three highly resolved light curves with cycle times below 100 s, obtained between January 25 and 30, 2007, to investigate this feature. Substructure on a timescale of several minutes is clearly identifiable, as demonstrated for instance in Fig. 4. After subtracting a strongly smoothed light curve to remove the low-frequency variations, a Lomb-Scargle analysis (Lomb 1976; Scargle 1982) was performed to search for periodicity. The periodogram in Fig. 5 shows the typical broad maxima, which could be associated with quasi-periodic flickering, but may also arise from a superimposition of different patterns. To exclude false positives, the influence of aperiodic, random brightness variations has to be considered. Such low-frequency flickering or "red noise" is caused by unsteady mass transfer and has to be distinguished from the more regular, quasi-periodic oscillations, excited by larger blobs in the accretion stream. To reproduce the red-noise and window effects in the observations, the observed power spectral density (PSD) is fitted with a power law over frequency, and light curves with similar mean count rate, standard deviation, and PSD shape are simulated on the time grid of the observed data. We adopt the Monte Carlo approach of Benlloch et al. (2001), which they use in the search for QPOs from active galactic nuclei, and determine confidence levels from the periodograms of 10000 fake light curves with the same power-law-shaped PSD. The corresponding levels of $99.9 \%, 99 \%$, and $95 \%$ significance are overplotted in Fig. 5. At two periods around $8 \mathrm{~min}$ and $5 \mathrm{~min}$, the power spectrum exceeds $99.9 \%$ significance, so the features may be considered to be real. The QPO frequencies found by Schwarz et al. (1998) are not significant at the $99.9 \%$ level.

No low-frequency periodicity was found in the XMM-Newton ultraviolet and soft X-ray light curves. All spikes in the periodogram lie well below the $90 \%$ confidence level. Rapid variations in the soft X-ray light curves are obvious, but no regular or periodic pattern is detectable.

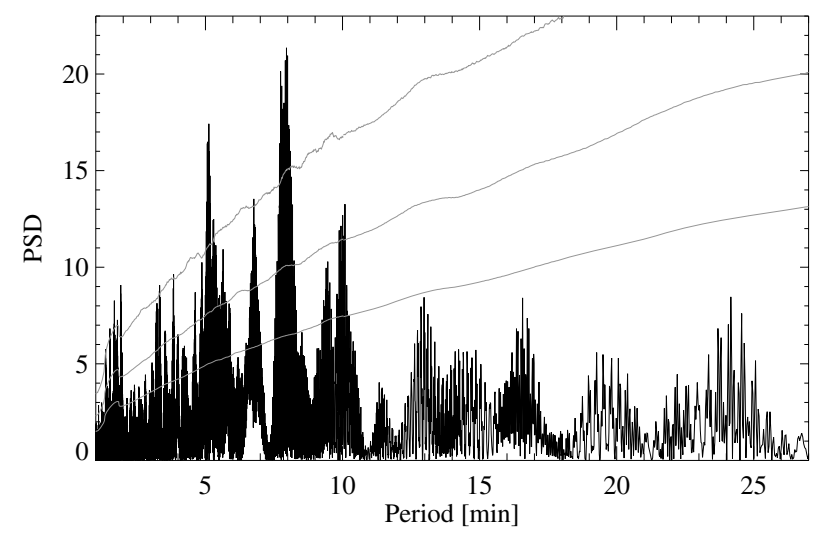

Fig. 5. Periodogram based on the highest time resolved $V$-band data from Jan. 2007. In grey, the 95\%, 99\%, and 99.9\% significance levels.

\section{X-ray spectroscopy}

With XMM-Newton, we obtained the first well resolved X-ray spectra with full orbital phase coverage of AI Tri. We used them to constrain the physical conditions and the structure of the accretion region on the white dwarf surface and of the post-shock accretion flow, and to compare the overall flux contributions from both origins. For this purpose, we analyzed the background-subtracted X-ray spectra by means of multicomponent models using XSPEC v12.5 (Arnaud 1996; Dorman et al. 2003). Initially, we modeled the time-averaged spectrum with single-temperature approaches for the soft and hard X-ray components. More complex and physically more relevant models were considered to improve the quality and the validity of the fits. In this context, we investigated separate fits to the spectra in the energy bands above and below $0.5 \mathrm{keV}$, and combined fits to the total spectrum. In a second step, we analyzed the average spectra of four characteristic phase intervals to constrain the accretion geometry. In all fits, we described the interstellar absorption using the TBNEW model ${ }^{2}$ with the abundances of Wilms et al. (2000) and the cross-sections of Verner \& Ferland (1996) and Verner et al. (1996). EPIC/pn and MOS data were modeled simultaneously. Treating the different instruments individually does not improve the fit quality, as tests via F-statistics confirmed. All errors are given at a $90 \%$ level of confidence in the following.

\subsection{Soft $X$-ray component (accretion spot)}

The flux contribution of the white dwarf was described by absorbed black bodies, mainly forming the spectral range below $0.5 \mathrm{keV}$ and originating in the accretion-heated stellar surface. The spectra exhibit deviations from a single black body that cannot be explained merely by uncertainties in the calibration. Within the accretion region, a wide range of temperatures is expected between the base of the accretion column and the unheated white-dwarf surface. To approximate this structure, we fitted multi-component black-body models, employing different Gaussian and exponential distributions of temperatures and emitting surface areas. They improved the fit statistics in the energy range below $0.6 \mathrm{keV}$ to $\chi_{\text {red }}^{2}=1.1$, compared to $\chi_{\text {red }}^{2}=1.3$ for a single absorbed black body. For the final fit over the whole energy range, we used black bodies with a Gaussian distribution

\footnotetext{
${ }^{2}$ Based on TBVARABS in XSPEC (Wilms et al. 2000), TBNEW provides refined cross-sections. See http://pulsar.sternwarte. uni-erlangen.de/wilms/research/tbabs/
} 
A\&A 516, A76 (2010)

Table 3. Results of the best XSPEC fits to the total and to the phase-resolved EPIC spectra of AI Tri.

\begin{tabular}{|c|c|c|c|c|c|c|c|c|c|c|c|}
\hline Model $^{a}$ & $\chi_{\mathrm{red}}^{2}$ & $\begin{array}{c}N_{\mathrm{H}, \mathrm{ISM}} \\
{\left[10^{20} \mathrm{~cm}^{-2}\right]}\end{array}$ & $\begin{array}{l}k T_{\mathrm{bb}} \\
{[\mathrm{eV}]}\end{array}$ & $\begin{array}{c}N_{\mathrm{H}, \mathrm{int}} \\
{\left[10^{23} \mathrm{~cm}^{-2}\right]}\end{array}$ & cover. & $\begin{array}{l}k T_{\mathrm{M}, \text { low }} \\
{[\mathrm{keV}]}\end{array}$ & $\begin{array}{c}k T_{\mathrm{M}, \text { high }} \\
{[\mathrm{keV}]}\end{array}$ & abund. & $\begin{array}{c}A_{\mathrm{bb}} \\
{\left[10^{6} \mathrm{~km}^{2}\right]}\end{array}$ & $\begin{array}{c}L_{\mathrm{bb}, \mathrm{bol}}{ }^{b} \\
{\left[10^{32} \mathrm{erg} \mathrm{s}^{-1}\right]}\end{array}$ & $\begin{array}{c}F_{\text {ХMM }} \\
{\left[10^{-12} \mathrm{erg} \mathrm{cm}^{-2} \mathrm{~s}^{-1}\right]}\end{array}$ \\
\hline \multicolumn{12}{|l|}{$2005 / 08 / 22$} \\
\hline MEKAL & 0.96 & $1.7_{-0.7}^{+0.8}$ & $43.0_{-3.0}^{+3.2}$ & $3.7_{-1.2}^{+2.4}$ & $0.76_{-0.12}^{+0.08}$ & - & $6.4_{-1.8}^{+2.6}$ & $0.8_{-0.4}^{+0.7}$ & $1.9_{-1.0}^{+2.5}$ & $2.2_{-0.9}^{+1.6}$ & $7.2_{-2.1}^{+3.5}$ \\
\hline 2MEKAL & 0.95 & $1.5_{-0.7}^{+0.9}$ & $43.7_{-3.0}^{+3.4}$ & $3.4_{-1.1}^{+1.7}$ & $0.75_{-0.07}^{+0.05}$ & $2.1_{-1.0}^{+2.1}$ & $9.1_{-3.0}^{+70}$ & $1.0_{-0.5}^{+1.0}$ & $1.6_{-0.8}^{+1.1}$ & $2.1_{-0.8}^{+1.5}$ & $7.0_{-2.0}^{+3.1}$ \\
\hline \multirow[t]{2}{*}{ 3MEKAL } & 0.94 & $1.6_{-0.6}^{+0.9}$ & $44.4_{-5.8}^{+4.3}$ & $3.3_{-1.2}^{+2.1}$ & $0.74_{-0.06}^{+0.08}$ & $0.06_{-0.02}^{+0.03}$ & & & & & \\
\hline & & & & & & $2.0_{-0.9}^{+3.5}$ & $9.2_{-2.8}^{+70}$ & $1.1_{-0.5}^{+1.3}$ & $1.1_{-0.7}^{+0.7}$ & $1.5_{-0.5}^{+1.5}$ & $8.4_{-2.8}^{+4.1}$ \\
\hline CEMEKL & 0.95 & $1.6_{-0.7}^{+0.8}$ & $43.7_{-3.1}^{+3.2}$ & $3.3_{-1.3}^{+2.3}$ & $0.72_{-0.12}^{+0.09}$ & - & $11.8_{-5.1}^{+13.3}$ & $1.2_{-0.7}^{+1.3}$ & $1.6_{-0.9}^{+2.1}$ & $1.9_{-0.5}^{-0.5}$ & $6.8_{-1.9}^{+3.3}$ \\
\hline MKCFLOW & 0.95 & $1.4_{-0.3}^{+0.4}$ & $44.5_{-0.5}^{+2.0}$ & $4.0_{-0.8}^{+1.0}$ & $0.68_{-0.06}^{+0.03}$ & $<0.003$ & $48.5_{-4.9}^{+17.4}$ & $1.7_{-0.8}^{+1.1}$ & $1.3_{-0.5}^{+0.4}$ & $2.0_{-0.8}^{+1.5}$ & $6.3_{-0.2}^{+0.2}$ \\
\hline accretion flow & 0.94 & $1.5_{-0.7}^{+0.8}$ & $43.9_{-3.2}^{+3.3}$ & $3.3_{-1.2}^{+2.5}$ & $0.72_{-0.12}^{+0.09}$ & $0.8_{-0.2}^{+0.4}$ & $20.0_{-6.1}^{+9.9}$ & $1.3_{-0.6}^{+1.0}$ & $1.6_{-0.8}^{+2.1}$ & $2.0_{-0.8}^{+1.5}$ & $6.6_{-1.8}^{+3.2}$ \\
\hline $\begin{array}{l}\text { Bright phase } 1 \\
\text { MEKAL }\end{array}$ & 0.95 & $1.9_{-0.9}^{+1.0}$ & $42.3_{-3.5}^{+3.7}$ & $1.8_{-0.7}^{+2.2}$ & $0.73_{-0.15}^{+0.10}$ & - & $4.6_{-1.3}^{+4.5}$ & $:=1.0$ & $4.9_{-3.0}^{+8.7}$ & $5.6_{-2.5}^{+5.5}$ & $16.4_{-6.1}^{+11.9}$ \\
\hline $\begin{array}{l}\text { Bright phase } 2 \\
\text { MEKAL }\end{array}$ & 1.03 & $1.8_{-1.3}^{+1.6}$ & $44.0_{-5.6}^{+6.1}$ & $1.6_{-0.8}^{+2.9}$ & $0.82_{-0.14}^{+0.11}$ & - & $4.7_{-2.2}^{+11.4}$ & $:=1.0$ & $2.0_{-1.5}^{+7.9}$ & $2.8_{-1.6}^{+5.5}$ & $8.0_{-3.7}^{+9.7}$ \\
\hline $\begin{array}{l}\text { Faint phase } \\
\text { MEKAL }\end{array}$ & 1.08 & $3.2_{-3.2}^{+4.3}$ & $25.1_{-19.2}^{+22.8}$ & $3.4_{-1.4}^{+3.5}$ & $0.85_{-0.07}^{+0.05}$ & - & $7.1_{-2.7}^{+4.8}$ & $:=1.0$ & $2.7_{-7.1}^{+7.1}$ & $2.0_{-1.1}^{+1.1}$ & $3.4_{-2.4}^{+8.7}$ \\
\hline $\begin{array}{l}\text { Minimum phas } \\
\text { MEKAL } \\
2005 / 08 / 15^{c}\end{array}$ & $e^{e}$ & $<3.0$ & $50.2_{-12.6}^{+17.8}$ & $14.7_{-6.7}^{+11.6}$ & 1.0 & - & $3.2_{-1.7}^{+9.9}$ & $:=1.0$ & $0.09_{-0.03}^{+1.09}$ & $0.15_{-0.47}^{+0.06}$ & $1.1_{-0.4}^{+1.1}$ \\
\hline $\begin{array}{l}\text { l005/08/15 } \\
\text { MEKAL }\end{array}$ & 1.08 & $3.6_{-0.6}^{+0.7}$ & $45.3_{-2.2}^{+2.2}$ & $15.6_{-10.9}^{+15.5}$ & 1.0 & - & $2.7_{-1.2}^{+16.0}$ & $:=1.0$ & $25.9_{-7.7}^{+13.2}$ & $38.0_{-8.3}^{+13.4}$ & $104_{-27}^{+42}$ \\
\hline
\end{tabular}

Notes. ${ }^{(a)}$ The soft component in all models is a multi-temperature black body with maximum temperature $k T_{\mathrm{bb}}{ }^{(b)}$ Bolometric black-body luminosities are determined as $L_{\mathrm{bb}, \mathrm{bol}}=2 \pi d^{2} F_{\mathrm{bb}}$, and the model fluxes by means of the CFLUX model. ${ }^{(c)}$ Simultaneous fit to EPIC and RGS data.

of temperatures and coupled emitting surface areas, fixing the minimum temperature at a typical white-dwarf surface temperature of $k T_{\mathrm{bb} \text {, low }}:=2 \mathrm{eV}(\sim 23000 \mathrm{~K})$. A maximum number of fifteen constituents were sufficient to reproduce a smooth temperature profile with small temperature steps, not exceeding $5 \mathrm{eV}$ between neighboring black-body components. The free parameters of the model were the temperature and the normalization of the hottest component. The model spectrum shows a shallower slope than a single black body. The highest X-ray flux arises from the hottest components with small effective areas, while the low-temperature black bodies covering larger surface areas contribute little to the total soft X-ray emission. The best-fit solution infers temperatures of as high as $k T_{\mathrm{bb} \text {,high }}=43.9_{-3.2}^{+3.3} \mathrm{eV}$, which corresponds to a characteristic temperature $k T_{\mathrm{bb}} \sim 39.5 \mathrm{eV}$ of a single black-body component. The hydrogen column absorption of $N_{\mathrm{H}, \mathrm{ISM}}=1.5_{-0.7}^{+0.8} \times 10^{20} \mathrm{~cm}^{-2}$ is on the same order as the Galactic $N_{\mathrm{H}}$ of up to $5 \times 10^{20} \mathrm{~cm}^{-2}$ towards AI Tri listed by Kalberla et al. (2005) and Dickey \& Lockman (1990).

\subsection{Hard X-ray component (post-shock accretion flow)}

To reproduce both the diffuse hot plasma and physical structure of the (post-)shock accretion column, we compared a number of different emission models (Table 3). Fits involving several independent plasma components provide a smaller $\chi^{2}$ than those with one mean temperature and, thus, demonstrate that emission regions at different temperatures influence the spectral shape. Multi-temperature models in XSPEC comprise a sum of MEKAL ${ }^{3}$ emission model components. We tested CEMEKL,

\footnotetext{
${ }^{3}$ MEKAL (e.g. Mewe et al. 1985; Liedahl et al. 1995) models include spectral continuum and element lines from a hot, optically thin plasma based on the assumptions of collisional ionization equilibrium and Maxwellian electron and ion energy distributions. Calculating the continuum radiation involves free-free, free-bound, and two-photon emission. Electron impact, radiative and dielectronic recombination,
}

where the emission measures are proportional to a power law over temperature, and the cooling flow MKCFLOW (Table 3). Using plasma models without considering the accretion physics, however, may lead to uncertain or erroneous bolometric flux estimates. Flux and luminosity ratios, used for investigating the energy balance (see Sect. 5), depend strongly on the model choice. We employ multi-temperature plasma models based on the emission region models of Fischer \& Beuermann (2001). They calculate the radiative transfer for bremsstrahlung and cyclotron radiation in a stationary two-fluid plasma. The local mass flow density is treated as the main free parameter and determines temperatures and shock height. For a characteristic primary mass of $M_{\mathrm{WD}}=0.6 M_{\odot}$, they derive temperature and density structures. We adopt these to account for the wide parameter range in the post-shock accretion column and to establish relations between the temperatures and the emission measures of several MEKAL components, assuming that optically thin conditions dominate. The element abundance, the emission measure, and the temperature of the coolest component are the free parameters for each $B-\dot{m}$ combination. In addition, a common absorption term is needed for the total plasma spectrum, which accounts for intrinsic absorption by material surrounding the emission region in the accretion column. We employ the partial-covering PCFABS and the solar metal abundance ratios of Grevesse et al. (2007) in the plasma models.

In a single-temperature MEKAL fit to the accretion-column spectrum of AI Tri, larger residuals remain around $1 \mathrm{keV}$ and between 3 and $5 \mathrm{keV}$. The multi-temperature plasma models describe the emission lines more precisely and improve the fit accuracy. The CEMEKL model clearly underestimates the continuum flux at energies above $7 \mathrm{keV}$, while MKCFLOW and accretion-flow models yield similar parameters. Closest approximation of the spectral lines is achieved when using the

and inner-shell excitation and ionization contribute to the line transitions. 
I. Traulsen et al.: X-ray spectroscopy and photometry of AI Tri with XMM-Newton

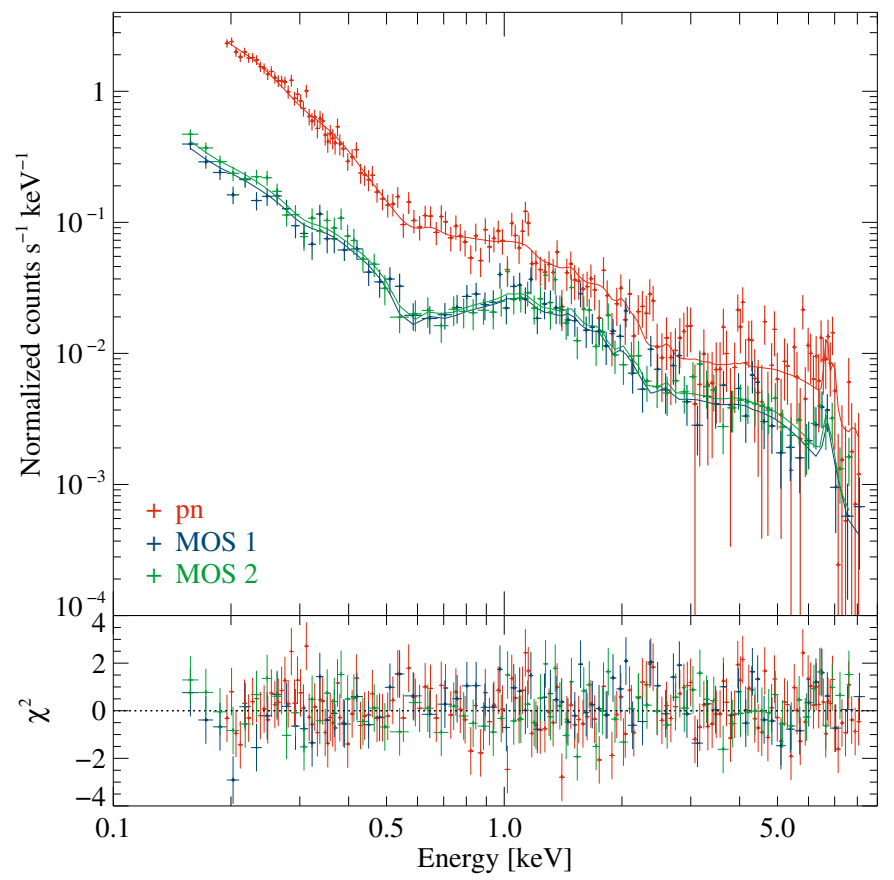

Fig. 6. EPIC spectra extracted from the $20 \mathrm{ks}$ exposure of AI Tri on August 22, 2005. The best-fit model consists of mildly absorbed black bodies, combined with partially absorbed multi-MEKAL plasma emission.

multi-MEKAL accretion-flow spectrum with the temperature structure following the models of Fischer \& Beuermann (2001, "accretion flow" model in Table 3). The magnetic field strength was fixed at $B=40 \mathrm{MG}$, referring to the result of Schwarz et al. (1998). From fits with mass flow densities between $\dot{m}=0.01$ and $100 \mathrm{~g} \mathrm{~cm}^{-2} \mathrm{~s}^{-1}$, an average $\dot{m}=0.1 \mathrm{~g} \mathrm{~cm}^{-2} \mathrm{~s}^{-1}$ is derived. The best-fit solution infers a reduced $\chi_{\text {red }}^{2}=0.94$ at 318 degrees of freedom, plasma temperatures between $k T_{\text {MEKAL,low }}=$ $0.8_{-0.2}^{+0.4} \mathrm{keV}$ and $k T_{\text {MEKAL,high }}=20.0_{-6.1}^{+9.9} \mathrm{keV}$, and $1.3_{-0.6}^{+1.0}$ times the solar abundances (Fig. 6).

Neither of the tested plasma models completely reproduces the spectral slope at energies above $4 \mathrm{keV}$. The deviations may be caused by remaining unresolved emission lines, which require a modified temperature structure or metal abundances. Alternatively, reflection of the continuum from the white-dwarf surface may harden the spectrum for energies above several $\mathrm{keV}$, as Done et al. (1995) expound for the GINGA spectra of EF Eri. The residuals around $1 \mathrm{keV}$ can indicate photoionization effects. An additional warm absorber model component such as ABSORI (Done et al. 1992; Zdziarski et al. 1995) or XSTAR (Kallman \& Bautista 2001; García et al. 2005) accounts more accurately for the spectral shape, but introduces too many free parameters.

\subsection{Phase-resolved modeling}

The 20 ks XMM-Newton exposure was divided into four phase intervals, characterized by the varying amount of soft X-ray emission and the associated hardness ratios (cf. Fig. 2). We extracted and modeled spectra for each of the intervals separately. Since the number of counts are lower than in the complete data set, we used absorbed multi-black body plus single MEKAL models. The element abundances were fixed to those of the solar distribution, which is approximately the orbital mean.

During the two X-ray bright phases, the spectral parameters agree remarkably well. Both the black-body luminosity and

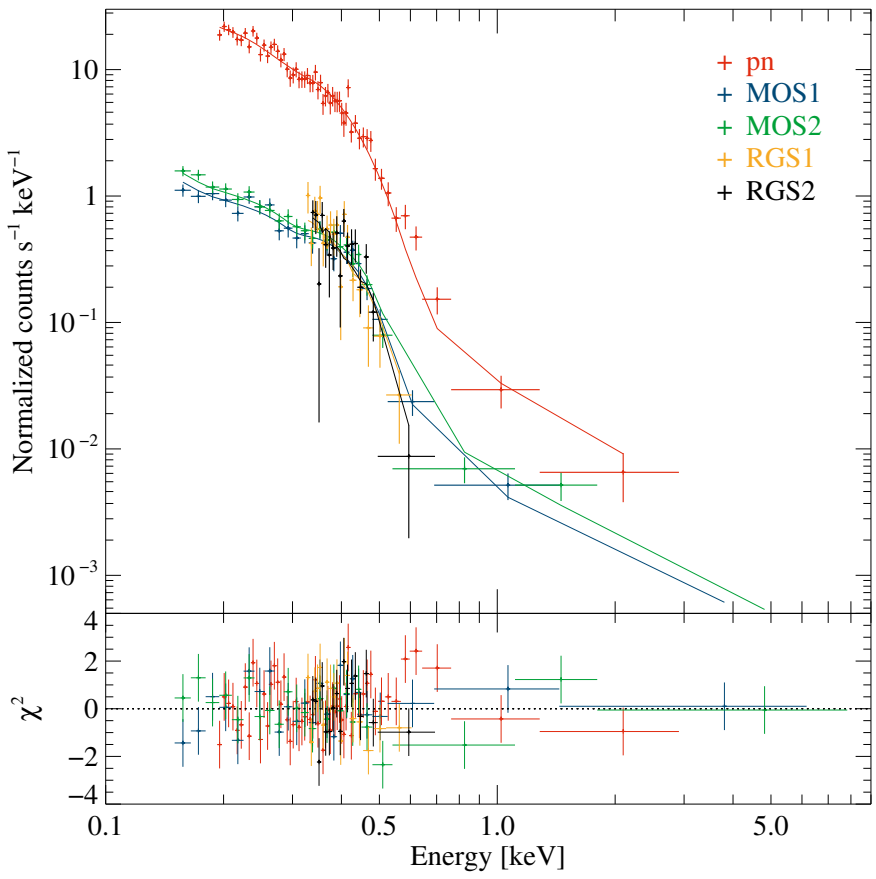

Fig. 7. Simultaneous fit to the XMM-Newton EPIC and RGS spectra on August 15, 2005 with the multi-temperature black-body plus single absorbed MEKAL model, as described in the text.

emitting surface area exceed the values derived from the fits to the total spectrum, while the contribution of the plasma component remains nearly constant. Owing to the higher background flux during the second bright phase, the fit is affected by larger uncertainties in the mean plasma temperature. On the whole, the two bright-phase spectra are identical within the error bars.

Apart from an intermittent soft flare before $\varphi_{\text {phot }}=1.0$, the soft X-ray count rate is consistent with zero during the faint phase. The hardness ratio reaches a mean value of $H^{\text {faint }}=$ $+0.53 \pm 0.01$ and a maximum of one. The model temperatures and the intrinsic absorption overlap with the bright-phase values within the large error bars and may be considered to be constant. A low signal-to-noise caused by the high background count rate is the main source of the uncertainties in the fit.

During the soft minimum phase interval, the low count rate also leads to a poorer fit accuracy. The black-body flux diminishes, and the hardness ratio $\mathrm{HR}_{\text {softmin }}=-0.43 \pm 0.01$ is similar to the orbital mean of $\mathrm{HR}_{\text {mean }}=-0.43 \pm 0.09$. In parallel, the intrinsic absorption increases substantially to $N_{\mathrm{H}, \text { int }}=$ $1.5_{-0.7}^{+1.2} \times 10^{24} \mathrm{~cm}^{-2}$, and the MEKAL norm rises, such that the plasma component contributes more significantly to the total $\mathrm{X}$-ray flux within the XMM-Newton energy range.

\subsection{XMM-Newton data on August 15, 2005}

From the 1.1 to 5 ks XMM-Newton observation on August 15, 2005, EPIC and RGS first order spectra were extracted and fitted simultaneously (Fig. 7). Since most of the signal was detected at energies below $1 \mathrm{keV}(\mathrm{HR}=-0.94 \pm 0.06)$ and the hard X-ray component almost vanished, we chose an absorbed multi-temperature black-body model plus a single MEKAL with fixed solar element abundance. The black-body parameters differ only marginally from the best fit to the $20 \mathrm{ks}$ exposure on August 22, 2005. The source flux estimated from the XSPEC fits, however, was at least a factor of ten higher than that during the $20 \mathrm{ks}$ exposure one week later. The significant increase in the 


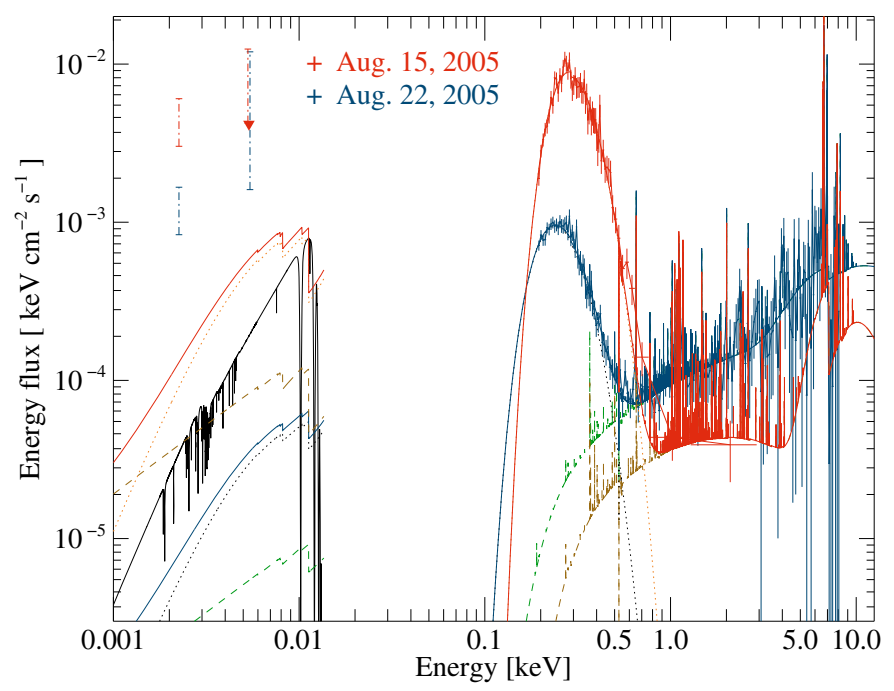

Fig. 8. Spectral energy distribution of AI Tri. To the right, EPIC/pn spectra from both the XMM-Newton pointings on August 15 and 22, 2005. To the left, the corresponding OM/UVM2 spectral points and the $V$-band observations on August 17 and 29, respectively, each with orbital minimum and maximum flux. On August 15, the UV minimum flux is unknown for lack of phase coverage. Overplotted are the best-fit models (solid lines) as described in the text, their individual components absorbed black body (dotted) and MEKAL (dashed) for each observation, and a non-LTE white-dwarf model spectrum at $T_{\text {eff }}=20000 \mathrm{~K}$ (black).

flux was caused mainly by the dominant soft X-ray component and a larger emitting surface area.

\subsection{UV flux}

To extend the spectral energy distribution coverage, we converted photometric measurements obtained at ultraviolet and optical wavelengths into energy flux units. The total OM/UVM2 flux in the range between $2050 \AA$ and $2450 \AA$ was calculated from the corrected count rate using the conversion factor from white dwarf standards, where 1 count s$^{-1}$ is equivalent to $2.2 \times 10^{-15} \mathrm{erg} \mathrm{cm}^{-2} \mathrm{~s}^{-1} \AA^{-1}$. It is similar in both the observations. Figure 8 shows the spectral points from our OM/UVM2 and $V$-band photometry, the EPIC/pn spectra, and the best-fit black-body plus MEKAL models. To be able to compare with the flux contribution of the unheated primary, a hydrogen-helium non-LTE white-dwarf atmosphere spectrum was calculated using the modeling package NGRT (Werner \& Dreizler 1999), whose predicted flux can serve as an approximate lower limit to the low-energy emission of the system. We assume a distance of $d \sim 620$ pc (Schwarz et al. 1998) and a typical effective temperature of $T_{\text {eff }}=20000 \mathrm{~K}$ for the white dwarf (Fig. 8). The orbital means of optical and UV flux exceed the models by factors up to 40 at both observational epochs. This is not surprising, as other system components such as the accretion stream can contribute significantly to the optical and UV flux. It is also consistent with the optical spectra presented by Schwarz et al. (1998), in which no signatures of the white dwarf could be detected. The emitting surface area of the low-temperature model components may be, in addition, significantly larger than derived from the $\mathrm{X}$-ray spectra. The UV data points can thus be taken to represent an upper limit, which is not violated by the spectral components required to model the $\mathrm{X}$-ray range.

\section{On the soft-to-hard X-ray flux ratios}

Several polars have a distinctly higher luminosity at soft than at hard energies. The true fraction of systems exhibiting this "soft X-ray excess" continues to be a topic of discussion. The phenomenon is apparently connected to the scenario of inhomogeneous and time-dependent ("blobby") column accretion proposed by Kuijpers \& Pringle (1982) and developed further by Frank et al. (1988). The amount of the excess increases with magnetic field strength (Beuermann \& Schwope 1994; Ramsay et al. 1994). Beuermann \& Burwitz (1995) point out that at a high field strength $(B>30 \mathrm{MG})$ and a relatively low accretion rate, cyclotron emission rather than bremsstrahlung becomes the main cooling process, causing an enhanced soft X-ray flux. Mass flow rate, magnetic field strength, and white-dwarf mass thus regulate the energy balance in polars (cf. Fischer \& Beuermann 2001). Ramsay \& Cropper (2004) examine new XMM-Newton and re-calibrated ROSAT data. Considering geometrical effects and the influence of accretion blobs, they derive soft-to-hard ratios close to one for the majority of the observed systems and conclude that fewer polars than previously understood exhibit a notable soft X-ray excess. With its strong magnetic field and known high soft X-ray emission, AI Tri is a suitable target to investigate the potential connection between system parameters, flaring signals from blobby accretion, and flux ratio.

\subsection{Photometric signs of blobby accretion}

Rapid variations characterize the optical as well as the X-ray light curves and are most likely caused by inhomogeneous accretion. Prominent flares may occur, when dense blobs impinge on the white-dwarf atmosphere. In the X-ray light curves, individual accretion events cannot be identified, though their shapes are probably formed by superimposed emission from multiple blobby events. This differs from the situation for the purely blobby accreting long-period polar V1309 Ori, where Schwarz et al. (2005) distinguished single accretion flares. The possible quasi-periodic oscillations in the optical light curves (Sect. 3.4), which may arise from blob-excited oscillations of the shock front, are in addition indicative of inhomogeneous accretion. Shafter et al. (1995) also report on QPOs at multiple frequencies in optical light curves of V1309 Ori.

\subsection{Flux ratios from multi-temperature spectral modeling}

The soft-to-hard X-ray flux ratios can be derived from unabsorbed bolometric luminosities. The observed data, however, cover only part of the spectral energy distribution, so the total flux has to be extrapolated and thus strongly depends on the choice of the model and its assumptions. In particular, results from former ROSAT analyses are uncertain, since the hard X-ray component had to be approximated with bremsstrahlung and the temperature could not be constrained from the spectral fits.

Our multi-temperature black-body and MEKAL model fit to the 2005 August 22 XMM-Newton spectra yields the typical parameters of the high-energy distribution of CVs. Using the distance estimate of AI Tri (Schwarz et al. 1998) $d=$ $620 \pm 100 \mathrm{pc}$, it results in an unabsorbed black-body luminosity of $L_{\mathrm{bb}}=2.0_{-0.8}^{+1.5} \times 10^{32}(d / 620 \mathrm{pc})^{2} \mathrm{erg} \mathrm{s}^{-1}$ and an integrated X-ray flux $F_{\text {bol }}=1.3_{-0.3}^{+0.7} \times 10^{-11} \mathrm{erg} \mathrm{cm}^{-2} \mathrm{~s}^{-1}$. The flux in the soft $0.1-0.5 \mathrm{keV}$ energy band exceeds the hard $0.5-10.0 \mathrm{keV}$ flux during the whole orbital revolution. The bolometric fluxes of the individual unabsorbed model components lead to ratios of $F_{\mathrm{bb}} / F_{\text {MEKAL }} \gtrsim 2.1_{-0.8}^{+1.6}$ for the orbital mean and 
of $\left(F_{\mathrm{bb}} / F_{\text {MEKAL }}\right)_{\text {bright1 }} \gtrsim 5.7_{-2.5}^{+5.6}$ for the first bright phase. Owing to the partial-covering absorption term employed in the spectral fits, the plasma temperatures are lower than in models that do not account for intrinsic absorption. In particular, the spectral resolution and range of the ROSAT spectra of polars was not high enough to derive both a reliable bremsstrahlung temperature and the absorbing column density. Hence, the cooler plasma component contributes more significantly to the emission in the soft $\mathrm{X}$-ray regime, resulting in a lower ratio of the extrapolated fluxes of the black-body to the MEKAL components.

On August 15, 2005, the total integrated flux $F_{\text {bol }}=1.6_{-0.5}^{+0.8} \times$ $10^{-10} \mathrm{erg} \mathrm{cm}^{-2} \mathrm{~s}^{-1}$ was notably governed by an increased blackbody component, while the model flux in the hard band stays at a low level. Due to the low count rate above energies of $0.8 \mathrm{keV}$, extrapolating the model flux to a wider energy range would have produced unacceptably large uncertainties. We therefore used the flux ratio in the XMM-Newton bands, $F_{\text {soft }} / F_{\text {hard }}=130_{-60}^{+95}$, as a lower limit to the bolometric flux ratio. The black-body flux is remarkably higher than on August 22, indicating that the mass accretion rate is higher by a factor of more than one hundred. This may induce a stronger temporal and spatial inhomogeneity in the accretion stream, forcing more material into dense blobs. In consequence, the contribution of the hard X-ray emission will be lower than in states of lower accretion rates, producing a distinct change in the spectral shape as seen in Fig. 8. The pronounced flickering in the X-ray light curves supports the idea of blobby accretion playing a larger role at that time.

An extreme dominance of the soft X-ray component was also found in the XMM-Newton spectra of V1309 Ori with a bolometric flux ratio of several thousand (Schwarz et al. 2005) and of the short-period polar EU UMa with a luminosity ratio of 14-2800 (Ramsay et al. 2004). In the few systems lacking distinct soft X-ray emission, such as 2XMMp J131223.4+173659 (Vogel et al. 2008) and V2301 Oph (Ramsay \& Cropper 2007), the flux maximum of the reprocessed component appears to be shifted towards the EUV and UV regime. The same could apply to objects that show a detectable, but very weak soft component, such as EV UMa (Ramsay \& Cropper 2003). This interpretation implies that single black-body models, adjusted to the X-ray regime, generally give only a lower limit for the reprocessed emission from the accretion region and thus for the flux ratio. To quantify the soft X-ray excess, multi-temperature models are required not only to describe the emission from the post-shock accretion column but also to adequately take into account the soft X-ray emission from the accretion area on the white dwarf.

\section{Accretion geometry of the system}

Depending on the field topology, the magnetic flux density, and the ram pressure of the free-falling accretion stream, coupling of the accretion plasma onto the field line trajectories can occur at different locations within the binary reference frame, and the infalling material may be channeled into one or more accretion regions on the white-dwarf surface. Constraining the geometry and location of the active X-ray emitting accretion region(s) is important to understanding the origin of X-ray soft and hard components and their flux balance.

Prominent light curve features and phase-resolved spectral modeling may provide insight into the system geometry. Since it is crucial to know their orbital phasing, we convert the photometric into orbital phases as described in Sect. 3.1 and refer to these values in the following discussion, which is valid based on the assumption of synchronous rotation. These features are for instance the two bright phases in our X-ray light curves and the minimum in-between. The almost identical fits to the bright-phase spectra strongly indicate that the complete soft $\mathrm{X}$-ray emission originates in one and the same accretion region. The minima around $\varphi_{\text {orb }}=0.26$ and $0.81\left(\varphi_{\text {phot }}=0.45\right.$ and 0.0$)$ are pronounced mainly in the soft X-ray flux and may have their origin in a variety of mechanisms. These include a total or partial self-eclipse of the accretion region by the white dwarf, absorption in the accretion stream, or random mass-transfer variations on timescales of several hours. An occultation of the white dwarf by the secondary star can be excluded, since it would be expected to occur at inferior conjunction.

\subsection{Self-eclipse of the accretion region}

A self-eclipse occurs when the accretion region passes behind the limb of the white dwarf because of the stellar rotation. Such a feature was found in the X-ray/EUV light curves of several polars such as AR UMa (Szkody et al. 1999), MT Dra (Schwarz et al. 2002), VV Pup (Vennes et al. 1995). The X-ray spectra during phases of self-eclipse are characterized by a vanishing emitting area, at least of the soft X-ray component, while the intrinsic absorption remains nearly constant.

At a system inclination of $i \sim 70^{\circ}$ (Katajainen et al. 2001), a partial or total self-eclipse is plausible for AI Tri, if the colatitude of the accretion region is higher than $20^{\circ}$. Of the two phase intervals with reduced X-ray emission detected for AI Tri, the major faint phase exhibits the characteristic signatures of a self-eclipse, indicating that larger parts of the accretion region might disappear behind the horizon of the white dwarf between $\varphi_{\text {orb }}=0.61$ and $\varphi_{\text {orb }}=0.86$. During the $\varphi_{\text {orb }}=0.21-0.31$ interval, the hardness ratio is not higher than zero, and soft and hard X-radiation are emitted at a similar level. An eclipse of the accretion region, hence, is less likely to explain this phase.

\subsection{Stream absorption}

When the accretion column crosses the line of sight, it can obscure parts of the accretion region. This manifests itself as a sharp dip in the soft X-ray light curves with a typical duration of $\Delta \varphi \sim 0.1$ and as significantly enhanced absorption in the X-ray spectrum. Light curves and spectra of AI Tri display this behavior during the soft X-ray minimum around $\varphi_{\text {orb }}=0.26$. The partial-covering absorption term in the spectral models rises markedly; the hardness ratio of the light curves increases from HR $\sim-0.8$ to values around zero (Fig. 2). The short ingress and egress times and the duration $\Delta \varphi=0.1$ of the light curve dip are also consistent with an origin in stream absorption. The energy-dependent dip due to stream absorption and the broad faint phase in the X-ray light curves of AI Tri resemble the properties of the high-field system AR UMa (Szkody et al. 1999) and the two-pole accretor MT Dra (Schwarz et al. 2002). Their X-ray emission can be explained in the same way.

\subsection{A second possible scenario}

The faint phase might alternatively be interpreted as stream absorption and the X-ray light curve dip at $\varphi_{\text {orb }}=0.26$ as a selfeclipse of the accretion region. In consequence, the optical and the UV light curves would then be anti-correlated with the X-ray data, which would disagree with the standard picture of accretion in polars (e.g. King \& Lasota 1979; Lamb \& Masters 1979) in which the maximum flux in the UV range is seen when the accretion region passes the line of sight - just at the phase of the 


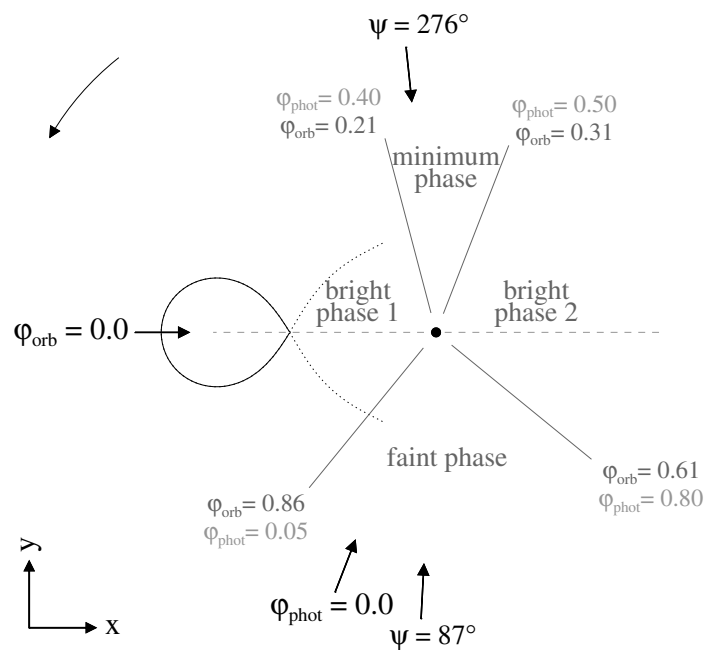

Fig. 9. Sketch of the binary geometry on the basis of the spectroscopic phasing derived by Schwarz et al. (1998), valid in the case of synchronous rotation, the Roche lobes being calculated for a mass ratio of $Q=M_{\mathrm{WD}}: M_{2}=1.5$. The individual phase intervals are marked to illustrate the formation of the most prominent light curve features.

stream eclipse. If the ultraviolet light curve, however, were dominated by the revolution of an extended accretion column, we may see its irradiated surface in anti-phase with the soft X-ray emission. Osborne et al. (1987) describe an anti-correlation of optical and soft X-ray flux for QQ Vul, another long-period polar, and consider a possible connection to accretion by means of dense filaments.

However, the length of the faint phase, the slow ingress, and the low intrinsic absorption do not agree well with the characteristics of a stream dip. The short soft minimum around $\varphi_{\text {orb }}=0.26$ cannot be identified in the former ROSAT light curves. A phase of self-eclipse, however, would be expected to recur on longer timescales. For both reasons, we consider this second scenario less likely.

\subsection{Orientation of the main accretion pole}

According to the standard picture of the accretion geometry in polars, described for example by Cropper (1988), the magnetic pole of the white dwarf onto which the major part of the accretion stream is directed is normally found between longitudes of $\psi=0^{\circ}$ and $90^{\circ}$. In the alternative interpretation of our X-ray light curves presented in Sect. 6.3, the accretion region of AI Tri would be visible from $\varphi_{\text {orb }}=0.31$ until $\varphi_{\text {orb }}=1.21$ and, thus, be located around $\psi=87^{\circ}$. In our favorite scenario (Sects. 6.1 and 6.2), on the other hand, the bright-phase emission is centered around $\varphi_{\text {orb }} \sim 0.23$, which corresponds to an unusual longitude $\psi=280^{\circ}$ of the dominating pole. But a standard geometry can also be preserved in this case, if the system rotates asynchronously. Asynchronous rotation cannot be excluded, as most of our observations (including the ROSAT and XMM-Newton data) trace solely the spin period. The information about both the orbital period and the phase shift between photometric and spectroscopic ephemeris is derived from the optical spectroscopy of Schwarz et al. (1998) in 1992/93, who report an upper limit on the order of $10^{-4}$ for the degree of synchronization. To place the accretion region of AI Tri within the first quadrant, the orbital phasing at the epoch of the XMM-Newton observations had to be shifted by $\Delta \varphi_{\text {orb }} \sim 0.2-0.5$. A phase shift of this order can be achieved by a small degree of asynchronism of at least
$\left|P_{\text {orb }}-P_{\text {spin }}\right| / P_{\text {orb }}=2-5 \times 10^{-5}$. This is even below the value of $0.28 \%$ that Staubert et al. (2003) derive for V1432 Aql, the polar with the slightest non-synchronous rotation determined up to now. Campbell \& Schwope (1999) indeed suggest that a significant number of polars with an undetected slight asynchronism may exist.

\subsection{The hard $X$-ray emission}

Low hard X-ray flux remains detectable throughout the orbital cycle. The emission may originate in an extended accretion region that is not totally eclipsed. Another possible explanation is a second accretion pole that is only visible in the high energy range and accretes at a low mass-flow rate. Episodes of accretion onto two poles with different hardness ratios were identified for example in AM Her (e.g. Matt et al. 2000), in MT Dra (Schwarz et al. 2002), and in BY Cam (Ramsay \& Cropper 2002), one of the few known asynchronous systems.

\subsection{Long-term variation}

The X-ray light curves obtained with ROSAT and XMM-Newton between January 1991 (Schwarz et al. 1998) and August 2005 (this work) do not completely repeat themselves. A pronounced faint phase before or around $\varphi_{\text {phot }}=0.0$ $\left(\varphi_{\text {orb }}=0.8\right)$, though, appears to be common to all X-ray light curves, which is consistent with the characteristics of a self-eclipse. It largely coincides with the UV and optical minima and with a peak in the linear polarization (Katajainen et al. 2001). The ROSAT HRI light curves taken between January 15 and February 5, 1998 are similar to the XMM-Newton light curves, but lack the deep soft $\mathrm{X}$-ray minimum around $\varphi_{\text {phot }}=0.45\left(\varphi_{\text {orb }}=0.26\right)$, identifiable in the XMM-Newton data on August 22, 2005.

The accretion scenario described above cannot explain all the phenomena in the XMM-Newton and archival ROSAT data. In particular, the emission during the second bright phase is lower than during the first one; and the count rate changes distinctly between the different X-ray observations. An expanded accretion curtain along the ballistic accretion stream might reduce the flux between $\varphi_{\text {orb }}=0.3$ and 0.7 , as confirmed in the case of HU Aqr by Schwope et al. (2001). Such an accretion curtain would account for the slightly enhanced absorption inferred by the spectral fits to the XMM-Newton data during the second bright phase, and be consistent with the finding that the narrow emission line components in the optical spectra are asymmetric (Schwarz et al. 1998). By more or less effective shielding, the accretion curtain could induce the other strong variations detected in the X-ray light curves. Schwope et al. (2001) report similar effects in the light curves and spectra of HU Aqr.

Other possible reasons for the long-term evolution of the light curves are switches between one-pole and two-pole accretion or a rearrangement of the accretion geometry. The location of the threading region, where the ionized matter in the accretion stream attaches itself to the magnetic field lines and is lifted off the orbital plane, depends on the mass accretion rate $\dot{M}$. A change in $\dot{M}$ will influence the stream trajectory and thus the region of impact onto the white dwarf at the footpoints of the magnetic field lines, whose spread determines the form, extent, and location of the accretion region. A large number of dense filaments in the stream in addition affects its structure. The phase shift of the irregular optical light curve minimum on August 17, 2005 could reflect this modified accretion 
geometry. The spectral fits to the 2005 August 15 XMM-Newton data indicate a highly increased mass accretion rate at this epoch (Sect. 5.2). Inhomogeneities and changes in the emission region, hence, are likely. The irregular mode of the $V$-band light curves on October/November 1992 (Schwarz et al. 1998) could be explained in the same way.

\section{Conclusions}

By analyzing our phase-resolved X-ray spectroscopy with XMM-Newton, we have confirmed a strong soft X-ray excess of AI Tri. Both the emission from the heated accretion spot and the emission from the post-shock accretion flow depart from single temperature models. From our multi-temperature approaches, we have found a bolometric flux ratio of $F_{\mathrm{bb}} / F_{\text {MEKAL }} \gtrsim 5.7_{-2.5}^{+5.6}$ between the two components during the bright phase of AI Tri on August 22, 2005. An even higher flux ratio of $F_{\text {soft }} / F_{\text {hard }} \gtrsim$ $130_{-60}^{+95}$ in the XMM-Newton energy bands was observed on August 15, 2005, when the total source flux was by a factor of at least ten higher. The distinct soft X-ray excess is probably related to inhomogeneous, blobby accretion as indicated by the high variability of the optical and X-ray flux on short timescales.

From the light-curve characteristics and the spectral variation in the intrinsic absorption with phase, we have inferred the most likely geometry of AI Tri. One main soft X-ray emitting accretion region undergoes a self-eclipse and a short period of accretion-stream absorption during the orbital revolution of the system. In this interpretation, the maxima of the UV and optical light curves occur at the moment that the accretion region passes the line of sight. The finding that hard X-ray emission is present at an almost constant, very low level over the whole orbital cycle, including the faint phase, may imply that a second accretion region with weak hard X-ray emission exists. Changes in the accretion geometry may occasionally occur, possibly in response to significant variations in the mass accretion rate. Signatures of these changes are the irregular light curves on October/November 1992 and on August 17, 2005, and the new minimum dip seen in the $V$-band light curves from November 2006 onward.

Assuming synchronous rotation of the white dwarf with the binary orbit, the main accretion region appears to be located around an unusual longitude of $\psi=280^{\circ}$. Since we extrapolated the spectroscopic ephemeris from data of Schwarz et al. (1998), obtained more than ten years before our multiwavelength observations, we cannot exclude there being a slightly asynchronous rotation of AI Tri within the accuracy reached. Future optical spectroscopy is necessary to clarify the ephemeris and to finally decide on the location of the accretion pole(s).

Acknowledgements. We thank F. V. Hessman and T. Nagel for carrying out the $V$-band observations at the MONET/North and Tübingen telescopes, and the referee for constructive remarks. I.T. gratefully acknowledges helpful discussions with S. Carpano, S. Fritz, D. deMartino, C. Mauche, and J. Wilms. This research is granted by DLR under project numbers 50 OR 0404 and 50 OR 0501.

\section{References}

Arnaud, K. A. 1996, in Astronomical Data Analysis Software and Systems V, ed. G. H. Jacoby, \& J. Barnes, ASP Conf. Ser., 101, 17

Benlloch, S., Wilms, J., Edelson, R., Yaqoob, T., \& Staubert, R. 2001, ApJ, 562, L121
Beuermann, K., \& Burwitz, V. 1995, in Cape Workshop on Magnetic Cataclysmic Variables, ed. D. A. H. Buckley, \& B. Warner, ASP Conf. Ser., 85,99

Beuermann, K., \& Schwope, A. D. 1994, in Interacting Binary Stars, ed. A. W. Shafter, ASP Conf. Ser., 56, 119

Beuermann, K., \& Thomas, H.-C. 1993, Adv. Space Res., 13, 115

Beuermann, K., Thomas, H., Reinsch, K., et al. 1999, A\&A, 347, 47

Beuermann, K., Burwitz, V., \& Rauch, T. 2006, A\&A, 458, 541

Campbell, C. G., \& Schwope, A. D. 1999, A\&A, 343, 132

Cropper, M. 1988, MNRAS, 231, 597

Dickey, J. M., \& Lockman, F. J. 1990, ARA\&A, 28, 215

Done, C., Mulchaey, J. S., Mushotzky, R. F., \& Arnaud, K. A. 1992, ApJ, 395, 275

Done, C., Osborne, J. P., \& Beardmore, A. P. 1995, MNRAS, 276, 483

Dorman, B., Arnaud, K. A., \& Gordon, C. A. 2003, in Bull. Am. Astron. Soc., 35,641

Fischer, A., \& Beuermann, K. 2001, A\&A, 373, 211

Frank, J., King, A. R., \& Lasota, J.-P. 1988, A\&A, 193, 113

García, J., Mendoza, C., Bautista, M. A., et al. 2005, ApJS, 158, 68

Grevesse, N., Asplund, M., \& Sauval, A. J. 2007, Space Sci. Rev., 130, 105

Kalberla, P. M. W., Burton, W. B., Hartmann, D., et al. 2005, A\&A, 440, 775

Kallman, T., \& Bautista, M. 2001, ApJS, 133, 221

Katajainen, S., Scaltriti, F., Piirola, V., Lehto, H. J., \& Anderlucci, E. 2001, A\&A, 372, 945

King, A. R., \& Lasota, J. P. 1979, MNRAS, 188, 653

Kuijpers, J., \& Pringle, J. E. 1982, A\&A, 114, L4

Lamb, D. Q., \& Masters, A. R. 1979, ApJ, 234, L117

Liedahl, D. A., Osterheld, A. L., \& Goldstein, W. H. 1995, ApJ, 438, L115

Lomb, N. R. 1976, Ap\&SS, 39, 447

Mateos, S., Saxton, R. D., Read, A. M., \& Sembay, S. 2009, A\&A, 496, 879

Matt, G., de Martino, D., Gänsicke, B. T., et al. 2000, A\&A, 358, 177

Mewe, R., Gronenschild, E. H. B. M., \& van den Oord, G. H. J. 1985, A\&AS, 62, 197

Osborne, J. P., Beuermann, K., Charles, P., et al. 1987, ApJ, 315, L123

Ramsay, G., \& Cropper, M. 2002, MNRAS, 334, 805

Ramsay, G., \& Cropper, M. 2003, MNRAS, 338, 219

Ramsay, G., \& Cropper, M. 2004, MNRAS, 347, 497

Ramsay, G., \& Cropper, M. 2007, MNRAS, 379, 1209

Ramsay, G., Mason, K. O., Cropper, M., Watson, M. G., \& Clayton, K. L. 1994, MNRAS, 270, 692

Ramsay, G., Cropper, M., Mason, K. O., Córdova, F. A., \& Priedhorsky, W. 2004, MNRAS, 347, 95

Scargle, J. D. 1982, ApJ, 263, 835

Schuh, S. L., Dreizler, S., Deetjen, J. L., \& Göhler, E. 2003, Balt. Astron., 12, 167

Schwarz, R. 1996, Diploma Thesis, Humboldt-Universität Berlin

Schwarz, R., Schwope, A. D., Beuermann, K., et al. 1998, A\&A, 338, 465

Schwarz, R., Greiner, J., Tovmassian, G. H., Zharikov, S. V., \& Wenzel, W. 2002, A\&A, 392, 505

Schwarz, R., Reinsch, K., Beuermann, K., \& Burwitz, V. 2005, A\&A, 442, 271

Schwope, A. D., Schwarz, R., Sirk, M., \& Howell, S. B. 2001, A\&A, 375, 419

Shafter, A. W., Reinsch, K., Beuermann, K., et al. 1995, ApJ, 443, 319

Standish, E. M. 1998, JPL IOM, 312.F-98-048

Staubert, R., Friedrich, S., Pottschmidt, K., et al. 2003, A\&A, 407, 987

Stuhlinger, M., Kirsch, M. G. F., Santos-Lleo, M., et al. 2008, Status of XMM-

Newton instrument cross-calibration with SAS v7.1, issue 5.0 edn., ESA, doc. No. XMM-SOC-CAL-TN-0052

Szkody, P., Vennes, S., Schmidt, G. D., et al. 1999, ApJ, 520, 841

Thomas, H., Beuermann, K., Reinsch, K., et al. 1998, A\&A, 335, 467

Vennes, S., Szkody, P., Sion, E. M., \& Long, K. S. 1995, ApJ, 445, 921

Verner, D. A., \& Ferland, G. J. 1996, ApJS, 103, 467

Verner, D. A., Ferland, G. J., Korista, K. T., \& Yakovlev, D. G. 1996, ApJ, 465, 487

Vogel, J., Byckling, K., Schwope, A., et al. 2008, A\&A, 485, 787

Werner, K., \& Dreizler, S. 1999, J. Comp. Appl. Math., 109, 65

Wilms, J., Allen, A., \& McCray, R. 2000, ApJ, 542, 914

Zdziarski, A. A., Johnson, W. N., Done, C., Smith, D., \& McNaron-Brown, K. 1995, ApJ, 438, L63

Zimmermann, H. U., Belloni, T., Izzo, C., Kahabka, P., \& Schwentker, O. 1993, in Astronomical Data Analysis Software and Systems II, ed. R. J. Hanisch, R. J. V. Brissenden, \& J. Barnes, ASP Conf. Ser., 52, 233 